\title{
Preface to the Special Issue of the 17th Chinese National Youth Conference on Catalysis (17th CNYCC)
}

Catalysis occurs when a catalyst forges a new reaction pathway that is unavailable to an uncatalyzed reaction. Catalysis is involved in almost $80 \%$ of chemical processes, and important applications include the synthesis of pharmaceutically active ingredients, production of petrochemicals and nitrogen fertilizers, the development of renewable energy and the reduction of pollution emission. In particular, green catalysis is at the heart of industrial and environmental challenges on energy, health, and sustainable development.

The Chinese National Youth Conference on Catalysis (CNYCC) was firstly initiated in 1987 at Dalian with fewer than 100 delegates, and currently the CNYCC has developed to be one of the most influential conferences on catalysis hosted by The Catalysis Society of China, in particular, CNYCC is the only conference dedicated to catalysis researchers under the age of 45. In August 2018, 17th CNYCC was held beside the beautiful Yellow River in Lanzhou jointly organized by Lanzhou Institute of Chemical Physics, Lanzhou University and Lanzhou Petrochemical Research Center, luckily it was the 30th anniversary of the CNYCC, over 1500 participants exchanged their recent developments and ideas on the topics including heterogeneous catalysis, homogeneous/molecular catalysis, biomass conversion, photo-/electrocatalysis, and catalytic mechanism study.

As a follow up of the 17th CNYCC, we organize this special issue, which contains 1 review, 2 communications, 9 original research papers. They cover research topics in single atom catalysis, conversion of $\mathrm{CO}_{2}$, energy catalysis, photo-/electrocatalysis, and in-situ characterization of catalyst fabrication and the evolution of catalyst structure. It is expected that this special collection of papers by young and active researchers will benefit to inspire and broaden the catalysis community.

We express our sincere appreciations to all the authors and reviewers for their significant contributions to this special issue. We also thank the editorial staff for their hardworking in editing and publishing this special issue.

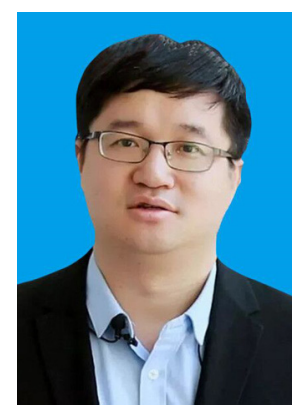

Prof. Xinchen Wang (Guest Editor)

State Key Laboratory of Photocatalysis on Energy and Environment,

College of Chemistry,

Fuzhou University

E-mail: xcwang@fzu.edu.cn

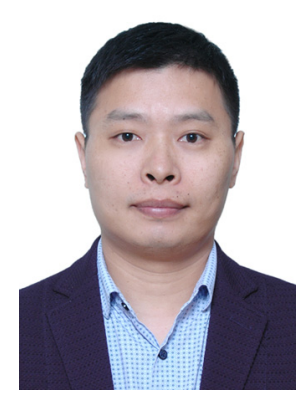

\section{Shuangyin Wang (Guest Editor)}

State Key Laboratory of

Chem/Bio-Sensing and Chemometrics College of Chemistry and Chemical Engineering, Hunan University

E-mail: shuangyinwang@hnu.edu.cn

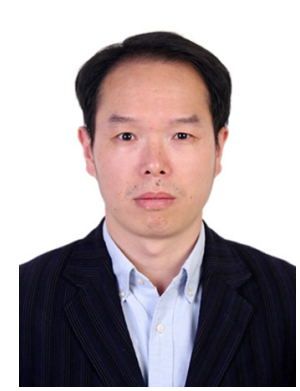

Fuwei Li (Guest Editor)

State Key Laboratory for Oxo Synthesis and Selective Oxidation, Lanzhou Institute of Chemical Physics, Chinese Academy of Sciences E-mail: fuweili@licp.cas.cn 